\title{
Motion artifact reduction in EEG recordings using multi-channel contact impedance measurements
}

\author{
Alexander Bertrand*, Vojkan Mihajlović ${ }^{\dagger}$, Bernard Grundlehner ${ }^{\dagger}$, Chris Van Hoof $^{\dagger}$, and Marc Moonen* \\ * KU Leuven, Dept. of Electrical Engineering ESAT/SCD, Kasteelpark Arenberg 10, B-3001 Leuven, Belgium \\ Email: \{alexander.bertrand, marc.moonen\}@esat.kuleuven.be \\ $\dagger$ Holst Centre / imec-nl, 5656 AE Eindhoven, The Netherlands \\ Email: \{vojkan.mihajlovic, bernard.grundlehner, chris.vanhoof\}@imec-nl.nl
}

\begin{abstract}
Dry-contact electrodes have paved the way for easyto-use electroencephalography (EEG) systems with minimal setup time, which are of particular interest in ambulatory as well as real-life environments. However, the presence of motion artifacts forms a major obstacle for such systems. In previous studies, it has been shown that continuous electrode-tissue impedance monitoring can be used to handle motion artifacts. In this paper, we demonstrate that the in-phase and quadrature components of the contact impedance provide complementary information that can be used to improve the prediction of motion artifacts. Furthermore, we demonstrate that the prediction of motion artifacts at one electrode can be further improved by also incorporating the impedance measurements at other electrodes. With this, we propose a motion artifact reduction algorithm based on a multi-channel linear prediction (MLP) filter. Although the MLP filter is not able to completely remove motion artifacts, a substantial reduction can indeed be achieved.
\end{abstract}

\section{INTRODUCTION}

Electroencephalography (EEG) is a non-invasive technique to monitor electrical activity of the brain by measuring voltage differences between electrodes that are attached to the scalp. To obtain high-quality EEG signals, the contact impedance between the skin and the electrodes should be sufficiently low. Therefore, traditional EEG recordings typically require the use of a conductive gel and proper skin preparation, which results in a long set-up time. Furthermore, since the gel dries out after a couple of hours, this approach is not suited for long-term EEG monitoring.

Recent advances in the area of biopotential sensors have lead to the development of so-called dry electrodes [1], which neither require skin preparation nor a conductive gel. Such electrodes drastically decrease the set-up time and improve the user comfort, but they are faced with a decreased signal quality compared to gel-based electrodes [2], [3]. Nevertheless, dry electrodes form an important alternative, especially outside a lab environment, e.g., for home-use, for long-term EEG monitoring, or for emergency situations where a fast deployment of an EEG system is required.

Motion artifacts are one of the most devastating disturbances in EEG signals recorded with dry electrodes. The proper handling of these motion artifacts is probably the most important challenge to be addressed in long-term EEG monitoring systems, where motion artifact detection and/or reduction will have to rely on sophisticated signal processing algorithms. Since motion artifacts are mainly caused by changes in the contact between the skin and the electrode, a continuous monitoring of the electrode-tissue contact impedance may help to estimate the EEG signal quality, or even to reduce the impact of the motion artifacts.

In [4], it was demonstrated that there is a significant correlation between the contact impedance magnitude and the EEG signal at a dry-contact electrode during specific movements. This indicates that continuous impedance measurements can indeed be used to detect and/or reduce motion artifacts. In this paper, we investigate whether the use of both in-phase and quadrature impedance components, rather than merely the impedance magnitude, increases this correlation. Furthermore, we demonstrate that the prediction of motion artifacts at one electrode can be further improved by also incorporating the impedance measurements at other electrodes. We then apply a multi-channel linear prediction (MLP) filter to these impedance measurements to predict motion artifact components and subtract these predictions from the corresponding EEG signals. We validate this MLP-based motion artifact reduction algorithm for multiple types of movements.

\section{Methods}

\section{A. Evaluation setup}

The wireless EEG prototype system used for the experiments in this paper consists of a rigid headset (see Fig. 1) with an integrated EEG signal acquisition system that allows to continuously measure the EEG signal and the contact impedance [5] at six $\mathrm{Ag} / \mathrm{AgCl}$ dry electrodes with pins (Biopac EL120). Four electrodes are positioned at $\mathrm{C} 3, \mathrm{C} 4, \mathrm{Cz}$, and $\mathrm{Pz}$, and the remaining two electrodes are positioned at the left and the right mastoid, which are used as ground and as reference, respectively. Both the in-phase $\left(z^{i}\right)$ and the quadrature $\left(z^{q}\right)$ component of the contact impedance $\left(z=z^{i}+j z^{q}\right)$ are measured at the electrodes $\mathrm{C} 3, \mathrm{C} 4, \mathrm{Cz}$, and $\mathrm{Pz}$, using a current of $20 \mathrm{nA}$ which is modulated with a square wave at $1024 \mathrm{~Hz}$. The EEG signal and the (demodulated) impedance signal are both sampled at $1024 \mathrm{~Hz}$ with a resolution of 12 bits. 

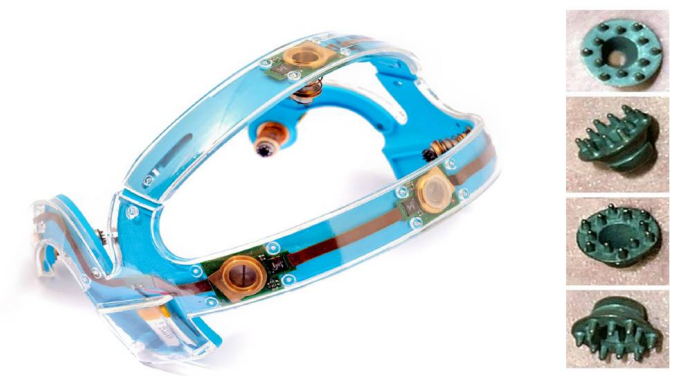

Fig. 1. 4-channel wireless EEG headset (imec) \& dry electrodes (Biopac)

\section{B. Evaluation protocol}

We included 6 test subjects ( 5 male and 1 female) in the evaluation. All participants signed an informed consent form before the start of the experiment. The EEG headset was mounted on the participant's head, and the signal quality was assessed by the experimenter based on a visual inspection using the EEG application software. In case a large impedance was observed and/or the EEG signal was too small, the headset was repositioned to overcome these unwanted effects. The experiment consisted of 5 sessions, each corresponding to a different types of movement:

1) Head movement in sagittal plane ('Nodding'): the subject was asked to slowly move his/her head up and down repeatedly during 1 minute while sitting on a chair.

2) Head movement in coronal plane ('Shaking'): the subject was asked to slowly tilt his/her head first to the left and then to the right repeatedly during 1 minute while sitting on a chair.

3) Standing up and sitting down ('Stand up/sit down'): the subject was asked to stand up from the chair and sit down again repeatedly during 1 minute.

4) Walking on the spot ('Walking'): the subject was asked to walk on the spot continuously for 1 minute.

5) Jumping on the spot ('Jumping'): the subject was asked to jump on the spot from a standing position during 1 minute. Every jump was followed by a period of approximately $2 \mathrm{~s}$ without any movement.

Each session is recorded two times: once with eyes open, and once with eyes closed. The latter is performed to induce significant $\alpha$-wave activity, such that this EEG phenomenon is more pronounced in the recordings. Each recording takes 3 minutes: one minute during which the subject was asked not to move, followed by one minute during which the subject was asked to perform one of the 5 movement types from the list above, again followed by one minute without movement.

\section{Preprocessing}

The same preprocessing steps were applied to each of the different signal types, i.e., the EEG signal and 3 impedance signals $\left(z^{i}, z^{q}\right.$ and $\left.|z|\right)$. All signals were first downsampled to $128 \mathrm{~Hz}$ using a proper anti-aliasing filter, after which they were high-pass filtered with a 600 tap linear-phase finite impulse response (FIR) filter with a cut-off frequency at $0.5 \mathrm{~Hz}$. A notch filter at $50 \mathrm{~Hz}$ has been applied to remove power-line
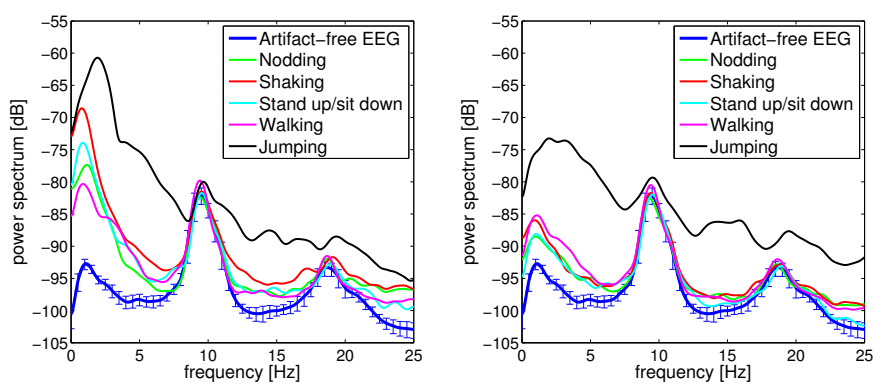

Fig. 2. Power spectrum of the EEG signal before (left) and after (right) MLP-based artifact reduction.

interference. From each recording, two 'artifact-free' signal segments of 55 seconds were extracted (one at the beginning and one at the end of the signal). The remaining signal segment of 70 seconds in the middle of the recording is from now on referred to as the 'artifact segment'. In total, $6 \times 5 \times 2=60$ of such artifact segments are obtained (6 subjects, 5 sessions, with two recordings per session).

\section{CORRELATION ANALYSIS}

The left plot in Fig. 2 shows the power spectrum of one EEG signal during different movements (here with eyes closed). The 'artifact-free' condition also shows the standard deviations computed from the power spectra of the 10 artifact-free signal segments of the particular subject. It is observed that most of the artifact energy is present in the low frequencies $(<5 \mathrm{~Hz})$.

To investigate to what extent the motion artifacts are reflected in the skin-electrode contact impedance signals, a canonical correlation analysis (CCA) has been applied [6]. Rather than examining the correlation between two individual signals, CCA allows to compute a correlation coefficient between two sets of signals, say, set A and set B. Specifically, CCA computes the correlation coefficient between two optimally chosen linear combinations of the signals in set A and of the signals in set B, respectively (we refer to [6] for further details).

Let $d_{k}$ denote the EEG signal at electrode $k$, and let $z_{k}^{i}$, $z_{k}^{q}$ and $\left|z_{k}\right|$ denote the in-phase component, the quadrature component and the magnitude of the impedance signal at electrode $k$, respectively (assume $k=1 \ldots K$, where $K=4$ in our setup). We define the 3 -dimensional local impedance signal at electrode $k$ as

$$
\mathbf{z}_{k}=\left[\begin{array}{lll}
z_{k}^{i} & \left.z_{k}^{q}\left|z_{k}\right|\right]^{T}
\end{array}\right.
$$

where $T$ denotes the matrix/vector transpose operator. We also define the stacked $3 K$-dimensional signal that contains the impedance signals of all electrodes:

$$
\mathbf{z}=\left[\begin{array}{llll}
\mathbf{z}_{1}^{T} & \mathbf{z}_{2}^{T} & \ldots & \mathbf{z}_{K}^{T}
\end{array}\right]^{T} .
$$

For the analysis in this paper, set $A$ always consists of the EEG signal $d_{k}$ at electrode $k$, whereas set B consists of one or more impedance signals:

- Set $\mathbf{B} 1$ contains the signal $\left|z_{k}\right|$. This is the same setup as used in the correlation study presented in [4].

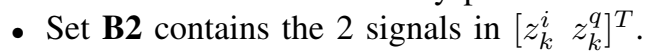



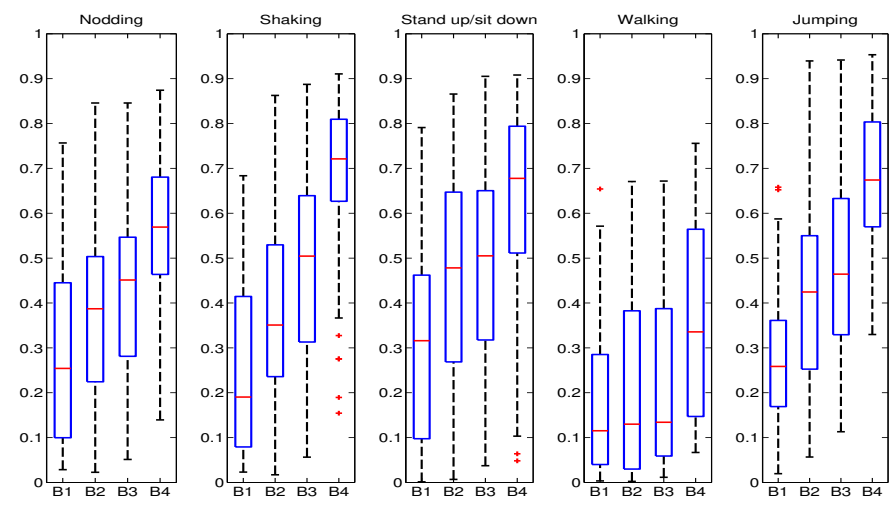

Fig. 3. Box plots of canonical correlation coefficients for different settings

- Set $\mathbf{B 3}$ contains the 3 signals in $\mathbf{z}_{k}$.

- Set $\mathbf{B 4}$ contains the 12 signals $\mathbf{z}$.

For each of the 5 movement types, we have performed a separate CCA on the 12 available artifact segments (6 subjects, with 2 recordings each, i.e., one with eyes open and one with eyes closed), resulting in 12 canonical correlation coefficients. The distribution of these coefficients is visualized by means of a box $\operatorname{plot}^{1}$ (for the different B-sets) in Fig. 3. Based on this figure, we can draw several conclusions:

- B1 vs. B2: Combining $z_{k}^{i}$ and $z_{k}^{q}$ into a single magnitude signal $\left|z_{k}\right|$ is suboptimal, i.e., the correlation improves if both $z_{k}^{i}$ and $z_{k}^{q}$ are used separately in the linear model.

- B2 vs. B3: Although the addition of $\left|z_{k}\right|$ in principle does not add extra information, it does improve the correlation. This is due to the fact that $\left|z_{k}\right|$ is a non-linear combination of $z_{k}^{i}$ and $z_{k}^{q}$. Since correlation only captures linear relationships, the addition of $\left|z_{k}\right|$ introduces an extra degree of freedom in the linear model, which will allow for a better modeling of motion artifacts.

- B3 vs. B4: The addition of the impedance signals of other electrodes substantially increases the correlation. This may be due to the fact that the electrodes are mounted in a rigid head set, and therefore the movement will influence all of the EEG and impedance signals in a similar fashion. Furthermore, there is a common motion artifact component between the different EEG signals induced by impedance changes at the reference electrode. Impedance signals that are well-correlated to this common component, can then help in modelling motion artifacts at other electrodes.

It should be noted that there is a large variation between the subjects, as demonstrated by the box plots in Fig. 3. This means that, for some subjects, there is not much correlation between the impedance signals and the EEG signals. This is probably due to the fact that the EEG headset does not fit equally well on each head. Furthermore, also the speed with which the movement is performed has an influence, i.e., fast movements typically show less correlation.

\footnotetext{
${ }^{1}$ The box plots were generated with the built-in Matlab command boxplot The red cross-shaped markers represent outliers.
}

\section{MLP-BASED MOTION ARTIFACT REDUCTION}

In the sequel, we assume an additive artifact model, i.e., the EEG signal at electrode $k$ can be written as

$$
d_{k}=\mathrm{EEG}_{k}+m_{k}
$$

where $m_{k}$ is the (motion) artifact due to a change in contact impedance, and where $\mathrm{EEG}_{k}$ is the EEG signal that would be observed with an ideal electrode with low and constant contact impedance. In the previous section, it has been demonstrated that there is often a significant correlation between the impedance signals and $m_{k}$. This motivates the use of an MLP filter to predict $m_{k}$ from the impedance signals.

We define the $3 L$-dimensional signal $\mathbf{y}_{k}[t]$, which consists of $L$ time lags of $\mathbf{z}_{k}[t]$, i.e.,

$$
\mathbf{y}_{k}[t]=\left[\mathbf{z}_{k}[t]^{T} \mathbf{z}_{k}[t-1]^{T} \ldots \mathbf{z}_{k}[t-L+1]^{T}\right]^{T}
$$

where we use the notation $s[t]$ to denote the sample of signal $s$ collected at sampling time $t$. We also define the stacked $3 K L$-dimensional signal

$$
\mathbf{y}[t]=\left[\begin{array}{llll}
\mathbf{y}_{1}[t]^{T} & \left.\mathbf{y}_{2}[t]^{T} \ldots \mathbf{y}_{K}[t]^{T}\right]^{T} .
\end{array}\right.
$$

The MLP filter is then defined as the multi-channel filter $\mathbf{w}$ that minimizes the mean squared error

$$
\min _{\mathbf{w}} E\left\{\left|m_{k}-\mathbf{w}^{T} \mathbf{y}\right|^{2}\right\}
$$

where $E\{\cdot\}$ denotes the expected value operator, and $\mathbf{w}^{T} \mathbf{y}$ is the linear prediction of $m_{k}$. Note that $m_{k}$ is an unknown signal, but since $\mathrm{EEG}_{k}$ is independent from both $\mathbf{y}$ and $m_{k}$, we can replace $m_{k}$ by $d_{k}$ in (6). The solution that minimizes (6) (with $m_{k}$ replaced with $d_{k}$ ) is given by [7]

$$
\mathbf{w}=\mathbf{R}_{y y}^{-1} \mathbf{r}_{y d_{k}}
$$

where $\mathbf{R}_{y y}=E\left\{\mathbf{y} \mathbf{y}^{T}\right\}$ and $\mathbf{r}_{y d_{k}}=E\left\{\mathbf{y} d_{k}\right\}$, which can be estimated by temporal averaging over $J$ samples, i.e.,

$$
\begin{aligned}
\mathbf{R}_{y y} & \approx \frac{1}{J} \sum_{j=0}^{J-1} \mathbf{y}[t-j] \mathbf{y}[t-j]^{T} \\
\mathbf{r}_{y d_{k}} & \approx \frac{1}{J} \sum_{j=0}^{J-1} \mathbf{y}[t-j] d_{k}[t-j] .
\end{aligned}
$$

Note that the widely-used least mean squares (LMS) and recursive least squares (RLS) adaptive filters [7], are adaptive implementations of the (M)LP filter described by (7)-(9).

In the experiments in this paper, we set $L=25$, which provides a good balance between computational complexity and prediction performance (note that $L$ depends on the sampling rate of the signals). We define $\bar{d}_{k}=d_{k}-\mathbf{w}^{T} \mathbf{y}$ as the cleaned-up EEG output signal. The right plot in Fig. 2 shows the power spectrum of $\bar{d}_{k}$ for the different movement types (for one subject). It is observed that the MLP filter preserves most of the EEG activity (this can be seen in the $\alpha$-wave band around $10 \mathrm{~Hz}$ ). The prediction of the motion artifact by the MLP filter is depicted in the center plot in Fig. 4 for the 'stand up/sit down' movement type. The resulting cleaned-up EEG 

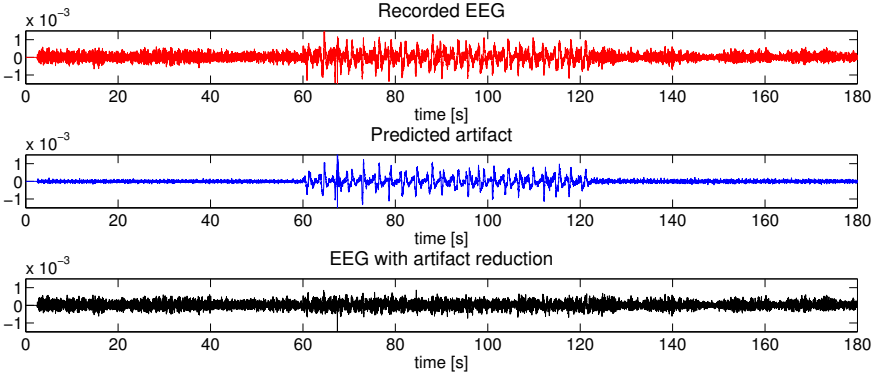

Fig. 4. Prediction of the motion artifact by the MLP filter during 'stand up/sit down' movement with eyes closed.

signal $\bar{d}_{k}$ is depicted in the bottom plot. It is noted that this is a well-chosen recording for which the MLP-based motion artifact reduction was relatively successful. To visualize the general performance over all subjects/movement types, we use two performance measures:

- The signal-to-artifact ratio (SAR), i.e., the variance $\sigma_{\text {eeg }}^{2}$ of the artifact-free EEG signal, divided by the variance $\sigma_{\text {art }}^{2}$ of the (residual) motion artifact. The former is estimated from $d_{k}$ during the two artifact-free segments, and the latter is estimated by subtracting $\sigma_{\text {eeg }}^{2}$ from the variance of the artifact segment of the same recording.

- The 'D-score', defined as the maximal distance between the empirical cumulative distribution function (ecdf) of the samples during the artifact-free segments and during the artifact segment, respectively. This is the test metric used in the so-called Kolmogorov-Smirnov test to determine whether two sample sets are generated by the same probability distribution.

Note that the higher the SAR, and the lower the D-score, the smaller the (residual) motion artifact component. The SARs and the D-scores are visualized by box plots in Fig. 5 and Fig. 6, respectively. We consider three different cases: (A) the original EEG signal $d_{k}$, (B) the cleaned-up EEG signal $\bar{d}_{k}$ using a 3-channel MLP filter on $\mathbf{y}_{k}$ (only electrode-specific impedance signals), and (C) the cleaned-up EEG signal using a 12-channel MLP filter based on y (all impedance signals).

We observe that including all impedance signals from all electrodes is beneficial for the motion artifact reduction. This is consistent with the correlation analysis in Section III, and can be explained by a similar discussion. We also again observe a large variance in the results, i.e., the performance of MLPbased motion artifact reduction is highly subject-dependent.

\section{CONCLUSIONS AND FUTURE WORK}

We have investigated the reduction of motion artifacts in EEG signals using continuous contact impedance monitoring. We have used CCA and MLP filtering to demonstrate that incorporating all three contact impedance signals (in-phase, quadrature and magnitude) substantially improves the prediction performance. Furthermore, the performance improves even more by incorporating all impedance signals from all electrodes. However, a significant residual artifact remains, as the MLP only captures linear dependencies. In future work, we will investigate the use of non-linear filtering techniques
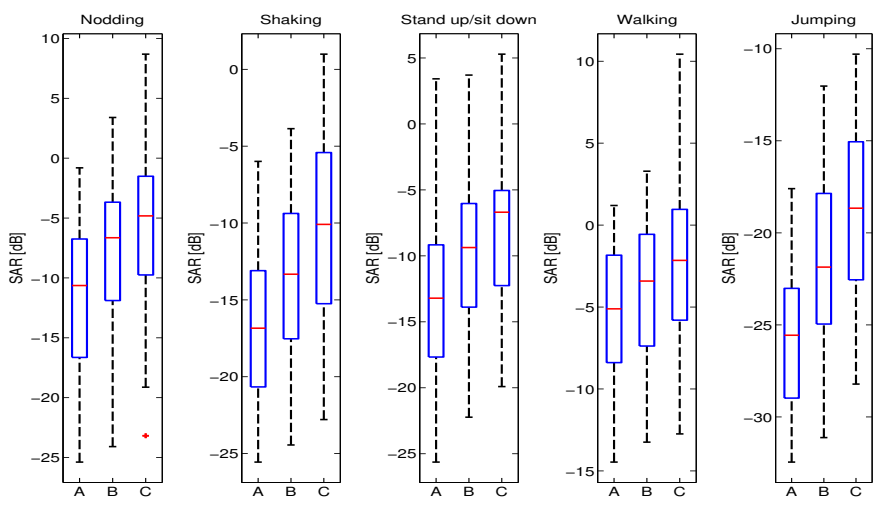

Fig. 5. SAR before motion artifact reduction (A), and after motion artifact reduction with 3-channel MLP (B) and full 12-channel MLP (C).
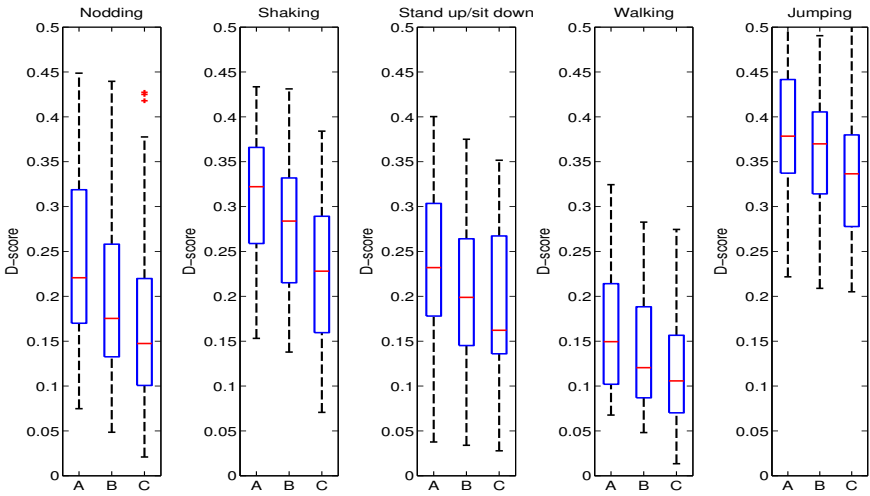

Fig. 6. D-scores before motion artifact reduction (A), and after motion artifact reduction with 3-channel MLP (B) and full 12-channel MLP (C).

to further improve the motion artifact prediction, as well as the use of adaptive filtering to allow for on-line processing.

\section{ACKNOWLEDGMENT}

The work of A. Bertrand was supported by a Postdoctoral Fellowship of the Research Foundation - Flanders (FWO). This work was conducted during Dr. Bertrand's visit to Holst Centre / imec-nl, as part of a collaboration with KU Leuven (ESAT), in the frame of KU Leuven Research Council CoE PFV/10/002 (OPTEC), Concerted Research Action GOA-MaNet, the Belgian Programme on Interuniversity Attraction Poles IUAP P7/23 (BESTCOM, 2012-2017), and Research Project FWO nr. G.0763.12 'Wireless acoustic sensor networks for extended auditory communication'.

\section{REFERENCES}

[1] Y. Chi, T.-P. Jung, and G. Cauwenberghs, "Dry-contact and noncontact biopotential electrodes: Methodological review," IEEE Reviews in Biomedical Engineering, vol. 3, pp. 106-119, 2010.

[2] G. Gargiulo, P. Bifulco, R. Calvo, M. Cesarelli, C. Jin, and A. van Schaik, "A mobile EEG system with dry electrodes," in IEEE Biomedical Circuits and Systems Conference (BioCAS), 2008, pp. 273-276.

[3] V. Mihajlovic, G. Garcia Molina, and J. Peuscher, "To what extent can dry and water-based EEG electrodes replace conductive gel ones?" in International Conference on Biomedical Engineering, 2011, pp. 14-26.

[4] V. Mihajlovic, H. Li, B. Grundlehner, J. Penders, and A. C. Schouten, "Investigating the impact of force and movements on impedance magnitude and EEG," in International Conference of the IEEE Engineering in Medicine and Biology Society (EMBC), 2013.

[5] S. Patki, B. Grundlehner, A. Verwegen, S. Mitra, J. Xu, A. Matsumoto, R. Yazicioglu, and J. Penders, "Wireless EEG system with real time impedance monitoring and active electrodes," in IEEE Biomedical Circuits and Systems Conference (BioCAS), 2012, pp. 108-111.

[6] D. Hardoon, S. Szedmak, and J. Shawe-Taylor, "Canonical correlation analysis: an overview with application to learning methods," Neural Computing, vol. 16, no. 12, pp. 2639-2664, 2004.

[7] S. Haykin, Adaptive Filter Theory, 3rd ed. Prentice Hall, 1996. 